\title{
Computational-chemical advances in heterogeneous catalysis
}

\author{
R.A. van Santen * \\ Schuit Institute of Catalysis, Eindhoven, University of Technology, Eindhoven, Netherlands
}

\begin{abstract}
A short overview is presented of current status of computational chemistry with respect to issues in heterogeneous catalysis. Applications to transition-metal- and solid-acid catalysis will be discussed.

Keywords: Quantum chemistry; Transition metals; Zeolites
\end{abstract}

\section{Introduction}

To experimental chemistry, quantum-chemistry has traditionally contributed in two ways:

(a) Interpretation of electronic or vibrational spectra.

(b) To formulate reactivity rules.

The main use of spectroscopy to the chemist is for structural information on compounds or complexes. The measurement of spectra implies an indirect procedure. On the basis of an assumed structure the spectrum has to be deduced. Comparison between theory and experiment makes structural identification possible. The main limitation in this process is the accuracy of the quantum-chemical calculation and size of chemical model that can be investigated. Current state of the art of quantum-chemistry is such that chemical model systems can be studied of direct relevance to surface chemistry.

As an example of such a procedure we will present computed spectra of the zeolitic Brønsted hydroxyl perturbed by chemisorbed acetonitrile

\footnotetext{
* Corresponding author.
}

[1]. This problem is of interest since it can only be solved when vibrational anharmonic coupling effects are completely included.

The Woodward-Hoffmann rules [2] provide an example of quantum-chemistry applied to chemical reactivity. Generalization of such semiempirical approaches to surface-chemical reactivity has been made [3]. The concept of surface orbital symmetry plays an important role.

The main advantage of current first-principle quantum-chemical approaches is that interaction energies can be accurately predicted. Hence deductions on the relative stability of chemisorbed complexes do not have to be based only on the analysis of the electronic orbital structure, but direct energetic information can be obtained. The results to be presented in the acetonitrile-zeolitic proton interaction also illustrate this important point. In this particular case it is used to deduce dynamic information on the vibrational motion of the proton. This relates to a third important application of quantum-chemical calculations:

(c) Computed potential energy curves can be used to deduce kinetic or dynamic constants of motion. 
Table 1

Computational quantum-chemical developments

Methods

Applications

a. (Quantum)-statistical mechanical calculations with quantum-chemically computed - predicted diffusion constants

potentials, transition state theory

b. Molecular dynamics simulations $(\Delta>5 \mathrm{kT})$ with accurate potentials

- kinetic modelling without lump-sum approximations

- solution chemistry

- solid state properties

c. Monte Carlo applications to overall reaction-rate predictions

- enzymes

d. Stochastic simulations of aggregation and crystallization

- aggregation of mesoporous systems

- predicted reaction-rate constants

e. Computer graphics

The current status is summarized in Table 1.

As long as the activation energy of an elementary reaction $\left(E_{\text {act }}\right)$ is larger than $5 \mathrm{kT}$, transition state reaction rate theory can be used to predict the reaction rate constant [4]. This will be illustrated for the reaction rate of dehydration of $\mathrm{CH}_{3} \mathrm{OH}$ to produce dimethyl ether catalysed by zeolitic protons [5].

Density functional theory [6] is a very useful first principle computational approach for electronic structure calculations on chemical models of catalytically relevant systems. Its usefulness derives from the accuracy of the predicted interaction energies and its usefulness to do calculations on large systems. The electron-correlation functional used allows for a significantly reduced computational cost for larger systems, than Hartree-Fock based methods, when they also include correlation energy correction terms.

All results to be discussed here will be based on density functional theory calculations. We will find in the study of methanol dehydration that even when small clusters are used, it will appear that adsorption energy and activation energy of dehydration agrees within $20 \mathrm{~kJ} / \mathrm{mol}$ with experiment. We will find a carbenium ion type transition state, very similar to the intermediate proposed in homogeneous acid catalysis.

The use of the cluster approximation to study chemisorption and surface chemical reactivity of transition-metal surfaces is less simple than for zeolites. It is now well known that the chemical reactivity of metal clusters is a strong function of cluster size [7]. We will illustrate this for the chemisorption of ethylene to small Pd clusters [8]. Chemisorption to a metal cluster leads to bond weakening of the $\mathrm{Pd}-\mathrm{Pd}$ bonds next to the chemisorption bond, which may lead reconstruction effects. It appears that reconstruction is also cluster size dependent and tends to decrease with increasing cluster size.

The accuracy of the density functional approach is such that it can also be applied to resolve also issues on mechanism. The dissociation energy activation barrier has been found for small molecules as chemisorbed $\mathrm{CO}$ or NO [9].

\section{Reactivity of zeolitic protons}

\subsection{Chemisorption of acetonitrile [1]}

To analyze the infrared spectrum of molecules chemisorbed to the proton in zeolites, it is essential to understand the consequences of anharmonic coupling effects. They may lead to Fermi-resonance coupling of modes in molecules, as well as between the proton modes [10]. When $\mathrm{CD}_{3} \mathrm{CN}$ instead of $\mathrm{CH}_{3} \mathrm{CN}$ Fermi-resonance effects in the probe molecule can be ignored and the shifts of the $\mathrm{CN}$ frequencies can be used to distinguish Brønsted acidic from Lewis acid sites.

The narrow proton absorption frequency at approximately $3650 \mathrm{~cm}^{-1}$ is replaced by a broad double peaked adsorption features around 2600 $\mathrm{cm}^{-1}$. The width of the adsorption band is due to strong coupling with weak $\mathrm{CH}_{3} \mathrm{CN}-\mathrm{H}$ vibration modes. The double peaked feature is due to Fermi- 


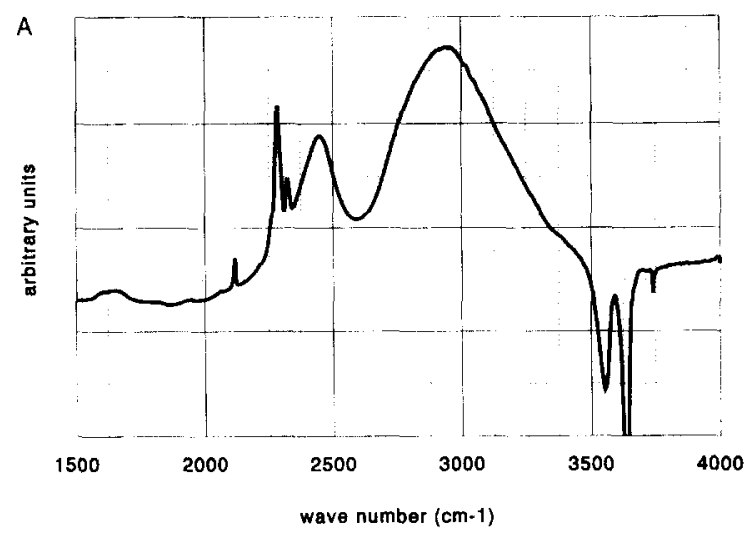

B

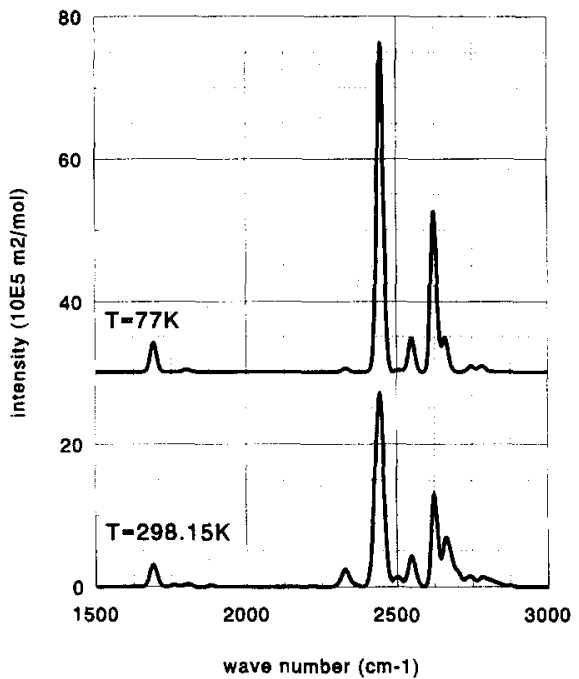

Fig. 1. (A) Experimental IR difference spectrum of $\mathrm{CD}_{3} \mathrm{CN}$ on $\mathrm{HY}$ (ref. [10]). (B) Computed IR spectra of acidic $\mathrm{OH}$ group with adsorbed $\mathrm{CH}_{3} \mathrm{CN}$ (ref. [1]).

resonance of the downward shifted $\mathrm{OH}$ stretch frequency with the upwards shifted lattice proton in-plane bending mode.

In Fig. 1 a comparison is made between an experimental $\mathrm{OH}$ spectrum and computed $\mathrm{OH}$ frequency spectrum of the chemisorption system. The spectra have been computed using a potential energy surface and mode coupling terms constructed from a four dimensional numerical fit of density functional theory electronic structure calculations. The three tetrahedral containing cluster $(\mathrm{Si} / \mathrm{Al}=2$ ), terminated by hydroxyl atoms gives an underestimation of the by proton-oxygen force constants. It is of the order of 5\%. This had to be adjusted to reproduce the experimental spectrum. It illustrates the issue of the dependence of $\mathrm{OH}$ bond strength on cluster size and zeolite composition. This problem has not yet been extensively studied quantum-chemically. Whereas trends can be analysed by variation of the $\mathrm{Si}-\mathrm{H}$ bonds that terminate the clusters, large cluster calculation are needed to solve this issue conclusively. According to our estimates [11] and that of others [12] clusters of at least 16 are needed to resolve this issue conclusively.

\subsection{Chemisorption and activation of $\mathrm{CH}_{3} \mathrm{OH}$ [5]}

Using a similar three tetrahedral cluster as applied to in the calculations on $\mathrm{CH}_{3} \mathrm{CN}$, we have studied the chemisorption and activation of $\mathrm{CH}_{3} \mathrm{OH}$.

Fig. $2 \mathrm{~A}$ shows the computed reaction energy diagram for the dehydration reaction of methanol, Fig. 2B shows the computed structures of reactant and product states of methanol as well as the corresponding transition states. The computations predict that methanol is adsorbed in a hydrogen bonded state, in which the acidic methanol proton is back-donated to a basic oxygen atom around the $\mathrm{Al}$ cation. The geometry agrees well with the computed and measured adsorption spectrum, as is illustrated by the data collected in Table 2 . The computed infrared spectrum has been obtained within the harmonic approximation. As a consequence the Fermi-resonance, present in the experimental spectrum, will not be reproduced by the calculation. Only one peak is found for the downwards shifted proton stretch frequency to a value of $\sim 2400 \mathrm{~cm}$ ! The activation energies and preexponents for the reaction rates of the different reaction steps in the dehydration reaction of methanol have been computed using transition state reaction rate theory. They indicate a significantly reduced mobility of the reacting molecule in the transition state. The computed heat of adsorption of end-on adsorbed $\mathrm{CH}_{3} \mathrm{OH}$ differs by $\sim 10 \mathrm{~kJ} /$ mol with the experimental value for $\mathrm{CH}_{3} \mathrm{OH}$ adsorbed in ZSM-5. The overall kinetics of the conversion reaction of methanol to dimethyl ether has an activation energy of $90 \mathrm{~kJ} / \mathrm{mol}$. This value 
A
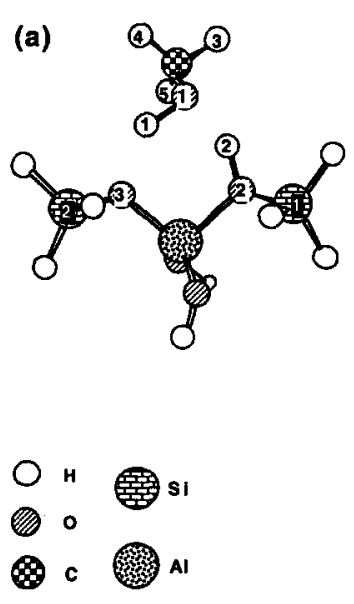

(d)

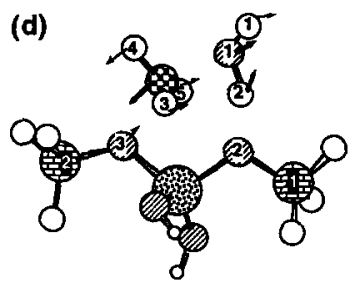

(b)
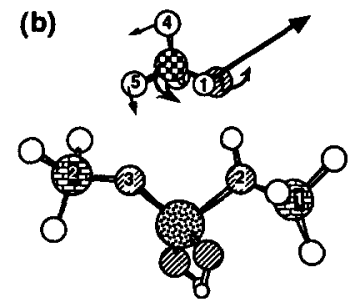

(c)

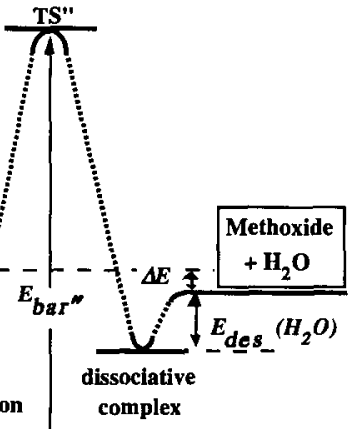

L. -
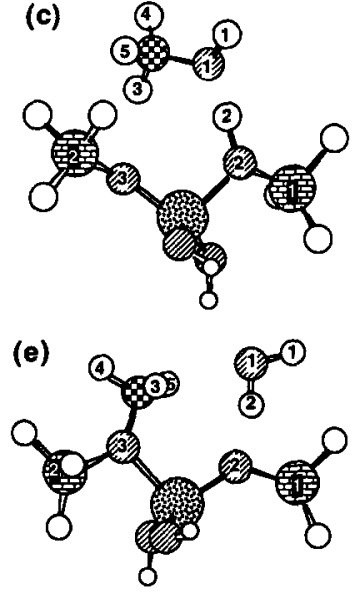

Fig. 2. (A) Adsorption $\left(E_{\mathrm{ads}}{ }^{\prime}, E_{\mathrm{ads}}^{\prime \prime}\right)$ and desorption $\left(E_{\mathrm{des}}\left(\mathrm{H}_{2} \mathrm{O}\right)\right.$ energies, electronic activation barriers including ZPE corrections $\left(E_{\mathrm{bar}}, E_{\mathrm{bar}}{ }^{\prime \prime}\right)$, Arrhenius activation energy with respect the 'end-on' adsorption complex and TS" ( $\left.E_{\text {act }}\right)$, (in $\mathrm{kJ} / \mathrm{mol}$ ) and effective Arrhenius pre-exponent $\left(A_{\text {eff }}^{\mathrm{TST}}\right.$, in $\left.\mathrm{m}^{3} \mathrm{~mol}^{-1} \mathrm{~s}^{-1}\right)$ for the reaction of $\mathrm{CH}_{3} \mathrm{OH}$ dehydration (ref. [5]) . (B) Dehydration of $\mathrm{CH}_{3} \mathrm{OH}$ : 'endon' (a) and 'side-on' (c) adsorption complexes, TS' (b), TS" (d) and dissociative complex (e) (ref. [5]).

is to be compared with $130 \mathrm{~kJ} / \mathrm{mol}$, predicted on the basis of the computed heats of adsorption and activation barriers, using the expression

$E_{\text {act }}($ eff $)=E_{\text {act }}($ diss $)-E_{\text {ads }}$

valid for a first order reaction, assuming the dehydration reaction to be rate-limiting. This also implies that the equilibration of the two adsorption modes of methanol is very fast. The computed steady state coverage of site-on bonded methanol is only $10^{-6}$ that of end-on bonded methanol.

It is important to note that the cluster geometry changes upon reaction with methanol. In the transition state, when the proton has been transferred to methanol, the $\mathrm{Al}-\mathrm{O}$ and $\mathrm{SiO}$ bond distances around the bridging oxygen from which the proton has been donated, contracts. In the dissociated state a methoxy species is formed on an oxygen atom around the Al centre previously unoccupied by a proton.

These cluster relaxation effects imply that the cluster embedded in the zeolite will stress the surrounding lattice, when protonation occurs. It has been demonstrated by the use of three-dimensional lattice calculations, that the zeolite lattice is quite flexible and the geometric changes as predicted in the cluster calculation are largely maintained also for the embedded situation [14].

Table 2

Calculated harmonic frequencies $\left(\mathrm{cm}^{-1}\right)$ for methanol adsorbed in the zeolitic cluster

\begin{tabular}{|c|c|c|c|c|c|}
\hline & \multicolumn{2}{|c|}{ 'side-on' } & \multicolumn{2}{|c|}{ 'end-on' } & \multirow{2}{*}{$\begin{array}{l}\text { Exp. } \\
{[13]}\end{array}$} \\
\hline & $\mathrm{AlOH}$ & $\mathrm{AlH}$ & $\mathrm{AlOH}$ & AlH & \\
\hline \multirow[t]{4}{*}{ OH stretch } & & & & & 354 \\
\hline & 3676 & 3692 & 3237 & 3245 & \\
\hline & 2911 & 2952 & & & 2900 \\
\hline & & & 2398 & 2416 & 2440 \\
\hline \multirow[t]{3}{*}{$\mathrm{CH}$ stretch } & 3120 & 3112 & 3086 & 3075 & 2993 \\
\hline & 3045 & 3046 & 3054 & 3058 & 2958 \\
\hline & 2970 & 2979 & 2972 & 2973 & 2856 \\
\hline \multirow[t]{4}{*}{$\mathrm{OH}$ bend } & & & & & 1687 \\
\hline & 1364 & 1351 & 1496 & 1483 & \\
\hline & 1255 & 1258 & 1372 & 1349 & \\
\hline & 1116 & 1108 & 1055 & 1040 & \\
\hline
\end{tabular}


The transition state of the dehydration of methanol, appears as an electrostatically stabilized $\mathrm{CH}_{3}^{+}$carbenium ion, weakly coordinated to $\mathrm{H}_{2} \mathrm{O}$ and the zeolite lattice. A very similar transition state has been computed for the dehydrogenation of $\mathrm{CH}_{4}$ [15]. Each reaction channel goes via a different transition state. For instance, the hydrogen-deuterium exchange reaction of $\mathrm{CH}_{4}$ proceeds by way of a carbonium ion type transition state, to be described as a $\mathrm{CH}_{5}^{+}$species, with nearly equivalent $\mathrm{CH}$ bonds $[15,16]$.

The examples chosen illustrate the detailed understanding that can be reached, due to application of the quantum-chemical cluster approach. For a more extensive review we refer to ref. [17].

Here we should mention the two important questions that a theory of zeolite catalysis has to answer:

1. How does activity relate to zeolite structure?

2. How does activity relate to zeolite composition?

For an answer to both questions the cluster approximation is not adequate. Using a molecular mechanics approach based on potentials deduced from quantum-chemical cluster calculations, we have concluded elsewhere, that differences in intrinsic acidity relate to the local relaxability of the framework around the reacting proton [14,18].

Hence methods are under development, that embed a quantum-chemically computed cluster into a classical mechanical system, that describes the zeolite lattice [19]. In this way the steric constraints and local compressibility can be taken in to account. An other important effect of the zeolite structure is found in the heat of adsorption of reactants and products as well as the steric constraints on accessibility to reacting molecules. For instance transition state selectivity has been claimed to be responsible for the high selectivity of ferrierite for the isomerisation of $n$-butene [20]. Docking procedures are available that enable the prediction of adsorption site accessibility [21]. Again the methods used are the Newton equations based on empirically determined potentials.
Related techniques are applied in molecular dynamics applications to compute the rate of diffusion. When barriers are low molecular dynamics can be successfully applied. Methods have also been developed to compute diffusion constants using transition state approaches for mobility of molecules with activation barriers higher than 5 $\mathrm{kT}$ [22]. The degree of success depends on the availability of classical potentials. This especially is an issue when the interaction with cations or polar molecules is to be studied.

In case zeolite particles are small and diffusional effects are small, it appears that differences in acidity of zeolites as catalytically probed by a conversion reaction can be determined to a large extend by differences in the heat of adsorption [23]. This has been demonstrated elegantly for the cracking of butane. A linear relation has been experimentally measured between overall activation energy and heat of adsorption under conditions where the reaction is first order in butane concentration. Concentration effects may affect the intrinsic acidity as well as adsorption energies and diffusion constants. Quantum chemical calculations involving larger rings of tetrahedra have demonstrated that changes in de-protonation energy can be understood in terms of changes in the covalent bonds within the zeolite framework and are short range [15]. The introduction of cations introduces locally a large electrostatic field, that requires a large cluster in order to be properly described. Large embedded clusters are needed to satisfactorily study changes in composition. The long range electrostatic effects can be accounted for by embedding the cluster in the electrostatic potentials as computed by the use of program as the CRYSTAL [11] routine.

\section{The coordination and activation of ethylene adsorbed to $P d$. clusters}

Computational studies on small clusters also show the importance of geometry changes when chemisorption occurs. 

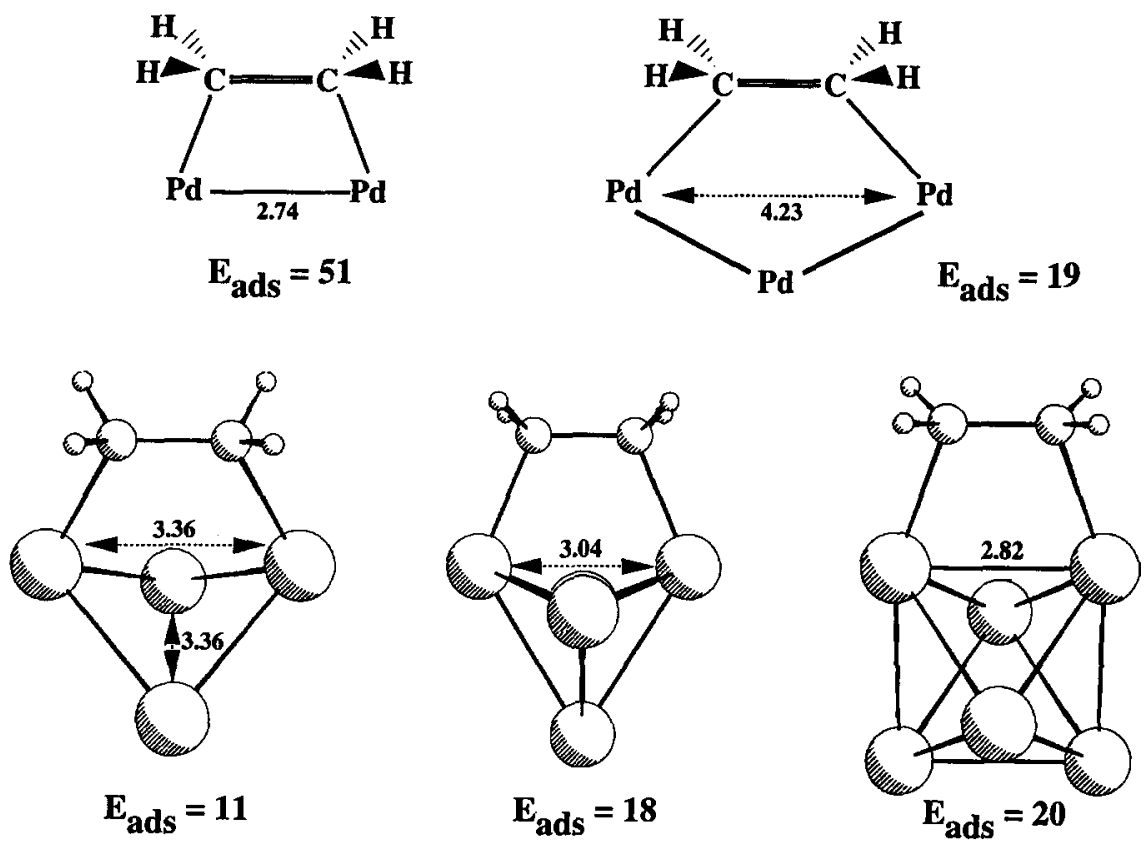

Fig. 3. Cluster size effect on the di- $\sigma$ adsorption mode of ethylene on palladium clusters $\operatorname{Pd}_{n}(n=1-6)$ (ref. [8]). Distances in $\AA$ and adsorption energies in $\mathrm{kcal} / \mathrm{mol}$.

In a very elegant study of chemisorption of hydrogen to Pd clusters of increasing size, Neurock et al. [24] found that changes in the bond lengths around an adsorption site become reduced when embedded into larger clusters with increasingly more atoms. Usually the geometric changes observed agree with predictions based on bond order conservation [25]. When the coordination number of an atom decreases, the bond lengths tend to decrease. These effects become less on larger clusters for two reasons. The first reason is the decreased interaction energy of the adsorbing atom with surface atoms that have an increasing number of neighbouring atoms [26]. The second is the constraint on atom displacement when in a lattice with a large elastic modules.

Earlier we studied and analysed [27] cluster reconstruction effects on small $\mathrm{Ni}_{3} \mathrm{~S}_{2}$ clusters with chemisorbed molecules.

Here we will illustrate the dramatic effects of bond relaxation using a recent study of the interaction of ethylene with Pd clusters of varying size (see Fig. 3) [8].

Note the large bond length and geometry changes and the changes in the interaction energy of ethylene.
Slab computational DFT techniques are available to model a crystal surface in two dimensions [28] with comparable accuracy as the cluster calculations that have been recently developed. With the advances in computational resources, the geometry optimization schemes that are indispensable for chemically accurate calculations currently absent will be implemented.

Whereas solid state approaches are indispensable for the study of overlayers and reconstruction effects, the cluster approach will remain useful for the study of reactivity at low coverage, because of the local nature of the disturbances that lead to relaxation of bond lengths and angles.

Elsewhere [26,27] we illustrated how quantum chemical calculations can be used to compute complete reaction cycles and how they can be used to decide on mechanism. Once complete catalytic reaction cycles have been computed, connection can be made with kinetics.

Also on transition metal clusters the activation energies and pre-exponents for elementary surface reaction rate constants have been computed using density functional theory computations. For $\mathrm{CO}$ and NO dissociation $\mathrm{Cu}$ cluster calculations [9] 
give the right ratio of pre-exponentials for dissociation versus desorption. An extensive analysis of the rate constants for the recombination of hydrogen with $\mathrm{CH}$ intermediates adsorbed to $\mathrm{Ni}$ and Co clusters has recently appeared [29].

With the knowledge of the reaction mechanism and capability to predict the reaction rate constants of elementary surface reaction steps one can formulate the kinetic equations and the expressions for the overall reaction rates.

This enables theory to close the pressure gap problems that exists between the kinetics of reaction systems at low pressure with kinetics of such systems at high pressure. As long as these changes are determined by differences in composition of the surface layer and changes in the rate limiting step.

Especially for the study of lateral interactions and reactions that require large surface ensembles the cluster approximation is critical. There is a need to do calculations on clusters in the range of 50-100 atoms and to do slab calculations involving the options of complete geometry optimization as well as to have efficient transition state searches. We expect these capabilities to develop rapidly in the near future.

\section{Conclusion}

In this paper we have briefly illustrated the status of current applied quantum chemistry in heterogeneous catalysis, using zeolite and metal catalysis as examples. As we discussed elsewhere [26] similar developments are ongoing in many additional areas of catalysis. Organometallic complexes and their transformations are widely studied in homogeneous catalysis [30]. Reactive oxide catalysts [31] and sulfides [27,32] are also subjects of extensive theoretical studies. A wealth of detailed microscopic information is becoming available, often difficult to obtain experimentally, but with an accuracy that is experimentally meaningful. Apart from standing computational problems, that mainly relate to the size of the systems to be studied, the main issue is choice of model systems. Crucial in this respect is access to experimental systems that correspond to the same systems as theoretically studies, notwithstanding their apparent remoteness from the working catalytic systems.

Computational techniques are developing in experimentalist tools. They provide an important means to bridge the gap of microscopic chemistry versus macroscopic kinetic modelling. An important area that will get more and more attention is the application of techniques to study the 'many' particle problems that appear in chemical kinetics of systems that are not ideally mixed or have energies that are due to the interaction of many particles.

In surface kinetics the problem arises when island formation or ordering effects occur in the surface layers. Lateral effects have to be understood as well as surface diffusion. To solve for the first the application of Monte Carlo techniques is being widely explored [33]. Molecular dynamics approaches to study diffusion or desorption from surfaces are also available [34]. Methods such as the generalized Langevin method have been applied to compute the rate of diffusion as well as desorption on transition metal surfaces [35]. For large activation barriers good agreement with transition reaction rate predictions has been found.

Also reactions in solutions require explicit incorporation of the interaction between many particles. The explicit consideration of water or other solved molecules in surface reactions has yet been little explored. Successful approaches in the homogeneous phase are however available [36].

Clearly the future will see extensions of current methods to systems of increasing complexity.

\section{References}

[1] E.L. Meier, R.A. van Santen and A.P.J. Jansen, J. Phys. Chem., (1995) in press

[2] R.B. Woodward and R. Hoffmann, Angew. Chem., Int. Ed. Engl, 8 (1969) 781.

[3] R. Hoffmann, Solids and Surfaces, VCH Weinheim, 1988; R.A van Santen, Theoretical Heterogeneous Catalysis, World Scientific, Singapore, 1991. 
[4] H.A. Kramers, Physica, 7 (1940) 284.

[5] S. Blaszkowsky and R.A. van Santen, J. Phys. Chem., 99 (3) (1995) 11728.

[6] see: J. Labanowski and J. Andrelin (Eds), Density Functional Methods in Chemistry, Springer, New York, 1991.

[7] see: G. Pacchioni, P.S. Bagus and F. Parmigiani (Eds.), Cluster Models for Surface and Bulk Phenomena, NATO ASI 375, 1991.

[8] A. Fahmi and R.A. van Santen, in preparation.

[9] M.A. van Daelen, Z.S. Li, J.M. Newman and R.A. van Santen, Chem. Phys. Lett., 226 (1994) 100.

[10] A.G. Pelmenchikov, R.A. van Santen, J. Jänchen and E. Meyer, J. Phys. Chem., 97 (1993) 11071.

[11] E.H. Teunissen, A.P.J. Jansen, R.A. Santen, R. Orlando and R. Dovesi, J. Chem. Phys., 101 (1994) 5865.

[12] H.V. Brand, L.A. Curtiss and L.E. Iton, J. Phys. Chem., 97 (1993) 12773.

[13] G. Witko, J.A. Lercher, M.W. Anderson and J. Klinowski, J. Chem. Soc., Faraday Trans., 86 (1990) 3039.

[14] G.J. Kramer, A.J.M. de Man and R.A. van Santen, J. Am. Chem. Soc., 113 (1991) 6435.

[15] S.R. Blaszkowki, A.P.J. Jansen, M.A.C. Nascimento and R.A. van Santen, J. Phys. Chem., 98 (1994) 11332.

[16] G.J. Kramer, R.A. van Santen, C.A. Emeis and A.K. Nowak, Nature 363 (1993) 529; V.B. Kazansky, I.N. Senchenya, M.V. Frach and R.A. van Santen, Catal. Lett., 27 (1994) 345.

[17] R.A. van Santen and G.J. Kramer, Chem. Rev.; 95 (3) (1995) 637.

[18] G.J. Kramer and R.A. van Santen, J. Am. Chem. Soc., 115 ( 1993) 2887.

[19] J. Sauer, Stud. Surf. Sci. Catal., 89 (1994) 2039.

[20] M.H. Mooiweer, K.P. de Jong, B. Kranshaar-Czarnebuc, W.H.J. Stork and B.C.H. Krutzen, Stud. Surf. Sci. Catal., 84 (1995) 2327.
[21] C.M. Freeman, C.R.A. Catlow, J.M. Thomas and S. Brode, Chem. Phys. Lett., 186 (1991) 137.

[22] R.L. June, A.T. Bell and D.N. Theoderou, J. Phys. Chem., 96 (1992) 1051; R.J. June, A.T. Bell and D.N. Theoderou, J. Phys. Chem., 94 (1990) 1508;

[23] W.O. Haag, Stud. Surf. Sci. Catal., 84 (1994) 1375.

[24] M. Neurock, G. Coulston and D. Dixon, in preparation.

[25] E.M. Shustorovich, Adv. Catal., 37 (1990) 101.

[26] R.A. van Santen and M. Neurock, Catal. Rev.-Sci. Eng., 37 (1995) 557.

[27] M. Neurock and R.A. van Santen, J. Am. Chem. Soc., 116 (1994) 4427.

[28] G. te Velde and E.J. Baerends, Phys. Rev. B, 44 (1991) 7888.

[29] H. Burghgraef, A.P.J. Jansen and R.A. van Santen, J. Chem. Phys., 101 (1994) 11012.

[30] A. Dedieu, in M. Gielen (Ed.), Topics in Organometallic Chemistry, Vol. 1, Freund, London, 1985, p. 1.

[31] M. Witko, J. Mol. Catal., 70 ( 1991) 277.

[32] T.S. Smit and K.H. Johnson, J. Mol. Catal., 91 (1994) 201222; T.S. Smit and K.H. Johnson, Chem. Phys. Lett., 212 (5) (1993) 525-533; T.S. Smit and K.H. Johnson, Catal. Lett., 28 (1994) 361-372; C. Rong and X. Qin, J. Mol. Catal., 64 (1991) 321-335; C. Rong, X. Qin and H. Jinglong, J. Mol. Catal., 75 ( 1992) 253-276. M.C. Zonnevijlle, R. Hoffman and S. Harris, Surf. Sci., 199 (1988) 320. J.K. Norskov, B.S. Claussen and H. Topsoe, Catal. Lett., 13 (1992) 1-8.

[33] V.P. Zhdanov, Elementary Physico-chemical Processes in Solid Surfaces, Plenum, New York, 1991.

[34] C.R.A. Catlow, C.M. Freeman, B. Vessal, S.M. Tomkinson and M. Lesli, J. Chem. Soc. Faraday Trans., 87 (1991) 411.

[35] J.C. Tully, G.H. Gilmer and M. Shugard, J. Chem. Phys., 71 (1979) 1630; A.P.J. Jansen, J. Chem. Phys., 94 (1991) 8444.

[36] W.L. Jorgensen, Adv. Chem. Phys. II, 70 (1988) 469. 